\title{
IONIC BEHAVIOR ASSESSMENT OF SURFACE-ACTIVE COMPOUNDS FROM CORN STEEP LIQUOR BY EXCHANGE RESINS
}

Rodríguez-López L., ${ }^{a}$ Rincón-Fontán M., ${ }^{a}$ Vecino X., ${ }^{b}$ Cruz J.M., ${ }^{a}$ and Moldes A. ${ }^{*}$

aChemical Engineering Department, University of Vigo, Campus As LagoasMarcosende, 36310 Vigo, Spain.

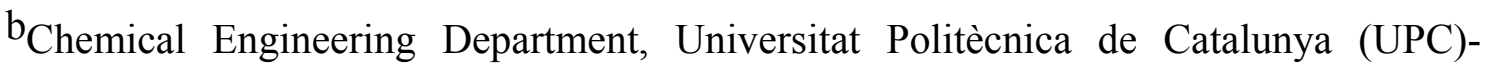
Barcelona TECH, Campus Diagonal-Besòs, Barcelona, Spain.

*Author corresponding: amoldes@uvigo.es 


\section{ABSTRACT}

Depending on their ionic nature, biosurfactants can be classified as nonionic, anionic, cationic or amphoteric. The ionic behavior of biosurfactants is an important characteristic that is going to delimit their industrial application. In this work a biosurfactant extract, obtained from corn steep liquor, was subjected to different extraction process with anionic or cationic resins in order to study its ionic behavior under different operational conditions through response surface methodology. The independent variables included in the study compromised the dilution rate of biosurfactant solution, the amount of cationic or anionic resin, and the extraction time; whereas the dependent variables studied consisted of the surface tension of biosurfactant aqueous solution after contacting with anionic or cationic resin. The results showed that biosurfactant extracted from corn steep liquor is amphoteric, both resins were able to entrap this biosurfactant, making it particularly suited for use in personal care preparations over sensitive skins.

Keywords: Biosurfactant; Surface activity; Interfacial science; Cationic surfactants; Anionic surfactants. 


\section{Introduction}

Generally, surfactants as well as biosurfactants can be cataloged based on the charged groups of the hydrophilic head as: nonionic, cationic, anionic and amphoteric. Nonionic surfactants do not have any charge groups over its head; whereas the head of an ionic surfactant posses a positive or negative net charge. If it is negative, the surfactant is named anionic and if the charge is positive, it is known as cationic. Moreover, other surfactants contain a head with two opposite charged groups, in which case they are named as amphoteric or zwitterion.

Those anionics are essential compounds in cleaning agents like shampoos, because of their excellent cleaning properties and high hair conditioning effects [1]. The most commonly used are alkyl sulphates, alkyl ethoxylate sulphates like sodium lauryl ether sulfate $[2,3]$ Cationic surfactants are quaternary ammonium compounds and they are mostly used for their disinfectant and preservative properties, as they have good bactericidal activity, what leads them to be used on skin for cleansing wounds or burns. Typically used cationic surfactants consist of cetrimide. Additionally, nonionic surfactants are less irritant than other anionic or cationic ones; the hydrophilic moiety of these synthetic compounds is composed by polyoxyethylene, polyoxypropylene or polyol derivatives; whereas their hydrophobic part contains saturated or unsaturated fatty acids or fatty alcohols. They have important properties as grease and oil removers and also can act as emulsifiers. The most commonly used nonionic surfactants are ethers of fatty alcohols. The other group is formed by the amphoteric. Those are very mild, making them particularly suited for personal care preparations over sensitive skins. Among the synthetic surfactants, amphoteric are considered less irritant than the cationic and anionic. Depending on the acidity or $\mathrm{pH}$ of formulations they can be anionic, cationic or nonionic. This is possible because they may contain two charged 
groups of different sign. These surfactants are characterized by their excellent dermatological properties, so they are frequently used in shampoos for kids and other cosmetic products. An example of this kind of surfactants is the cocamidopropyl betaine [3]. Thus, Corraza et al. [4] have compared the irritant and sensitizing potential, in 105 patients, of some synthetic surfactants, usually employed in the formulation of marketed synthetic surfactants, including anionic, cationic, amphoteric, and nonionic; and they found that irritation from surfactants contained in detergents is a frequent adverse reaction to cosmetics. Otherwise, it was observed that the most tolerated were the mild anionic and amphoteric surfactants.

Although the ionic properties of synthetic surfactants are well known, the ionic nature of most biosurfactants reported in the literature has not been explored yet. Biosurfactants are generally classified following their chemical structure and microbial origin [5]. However, for the inclusion of biosurfactants in industrial formulations it is mandatory to know their ionic nature, as it is going to delimit their potential application. In comparison with synthetic surfactants, biosurfactants are less irritating and more biocompatible [6].

Biosurfactants are composed by polymeric fractions of lipids, peptides and carbohydrates; structurally they usually contain a hydrophilic moiety, composed by acids, alcohols, peptide cations, or anions, mono-, di- or polysaccharides and a hydrophobic moiety, composed by unsaturated or saturated hydrocarbon chains or fatty acids [5]. Therefore, it is expected that they will be more biocompatible than the synthetic surfactants. However, the industrial application of biosurfactants is restricted by their low productivity, expensive downstream processing and lack of appropriate understanding of the bioreactor systems for their production. Thus, till now, only rhamnolipids and surfactin are commercially available. For this reason, it will be 
interesting to explore the extraction of biosurfactants from industrial streams like that obtained in the corn milling industry. Thus, in previous works, Vecino et al. [7] have extracted a lipopeptide biosurfactant from corn steep liquor that is able to reduce the surface tension of water in more than 30 units. This biosurfactant could compete with synthetic surfactants, in production cost, because it is produced spontaneously and it is not necessary the use of specific bioreactor systems for their production, as it is generated as a by-product. However, in order to define its field of application, it is needed to know the charge of this natural surfactant.

The aim of this work was to establish the ionic nature of a lipopeptide biosurfactant, extracted from corn steep liquor, using cationic and anionic resins, under different operational conditions. This study was carried out by applying an incomplete factorial design where the independent variables were: dilution rate applied to the biosurfactant solution $\left(X_{1}\right)$, amount of anionic or cationic resin $\left(X_{2}\right)$ and extraction time $\left(X_{3}\right)$; whereas the dependent variables were the surface tension achieved in the biosurfactant solution after contacting with the anionic or cationic resin corresponding with $Y_{1}$ and $Y_{2}$ respectively.

\section{Materials and methods}

\subsection{Extraction of biosurfactant from corn steep liquor (CSL)}

The biosurfactant was extracted from CSL (Santa Cruz Biotechnology, Lot L1813) using the protocol established in previous work [8]. Therefore CSL, containing 50\% of solids was diluted in water at $50 \mathrm{~g} / \mathrm{L}$, and extracted with chloroform (ratio CSL solution:chloroform 1:2) at $56^{\circ} \mathrm{C}$ during $60 \mathrm{~min}$. After that, chloroform was evaporated by vacuum distillation and the biosurfactant was dissolved in water up to reach its critical micelle concentration (CMC). The $\mathrm{CMC}$ of the biosurfactant was established by diluting the extract of biosurfactant in water. Below the $\mathrm{CMC}$, the concentration of 
biosurfactant is directly proportional to its capacity to reduce the surface tension of media. The solution of biosurfactant at the CMC was named BS.

The yield of the biosurfactant extraction was calculated by taking $2 \mathrm{~mL}$ of the solution containing the biosurfactant and drying it at $105^{\circ} \mathrm{C}$ for $24 \mathrm{~h}$. All measurements were carried out by triplicate.

\subsection{Surface activity determination}

The surface tension of solutions containing the biosurfactant extract was determined using a tensiometer with platimum plate (KRUSS K20 EasyDyne Tensiometer equipped with a $1.9 \mathrm{~cm}$ Wilhelmy platinum plate). Measurements were made in triplicate samples to increase the accuracy of the measurements.

\subsection{Biochemical characterization of the biosurfactant}

In order to determine the relative amount of fatty acids of the biosurfactant extract obtained, using the Fatty acid methyl esters (FAMEs) method, the sample was previously derivatized. For that, a total amount of $4.82 \mathrm{mg}$ of biosurfactant extract from CSL were diluted in $3 \mathrm{~mL}$ of $\mathrm{CHCl}_{3}: \mathrm{CH}_{3} \mathrm{OH}(1: 2, \mathrm{v} / \mathrm{v})$ and well mixed. After $15 \mathrm{~min}$ in darkness, a solid was formed, so the sample was centrifuged for $10 \mathrm{~min}$ at $4^{\circ} \mathrm{C}$ and 5000 rpm in order to separate it. This solid was extracted using $3.6 \mathrm{~mL}$ of $\mathrm{H}_{2} \mathrm{O}: \mathrm{CHCl}_{3}$ mixture 1:1 (v/v). The aqueous phase was eliminated and $50 \mu \mathrm{L}$ of internal standard (Heptadecanoic acid methyl ester $\mathrm{C} 17: 0)$ were added to the organic solution. The solvent was removed using a vacuum distillatory. After that, $2 \mathrm{~mL}$ of $\mathrm{HCl} 5 \%(\mathrm{w} / \mathrm{w})$ diluted in methanol were added to the vial, it was closed and heated to $85^{\circ} \mathrm{C}$ for $2.5 \mathrm{~h}$. The analysis was carried out using a GC-MS-MS on a Model Scion 451 GC (Bruker) equipped with a PTV 1019 universal capillary injector, coupled to a mass spectrometer, and controlled by Sistem Control Software. The FAMEs separation was performed on a DB-WAX column (30 m x $0.25 \mathrm{~mm}$ i.d. $\times 0.25 \mu \mathrm{m}$ film thickness). Helium was used as 
carrier gas with a flow rate of $1 \mathrm{~mL} / \mathrm{min}$, being the temperature of the injector inlet and the transfer line of the detector set at $240^{\circ} \mathrm{C}$. The mass spectra were obtained using a mass-selective detector under electron impact ionization at a voltage of $70 \mathrm{eV}$. Furthermore, the oven temperature was held initially at $50^{\circ} \mathrm{C}$ for $2 \mathrm{~min}$, then increased to $220^{\circ} \mathrm{C}$ at $4{ }^{\circ} \mathrm{C} / \mathrm{min}$ and finally kept at this temperature for $15 \mathrm{~min}$.

FAMEs were identified using a mass spectra library supplied with the GC-MS-MS system and by comparison of retention times and mass spectra of a FAMEs standard mix (Supelco 37 Component FAME Mix: $10 \mathrm{mg} / \mathrm{mL}$ of the FAME reference standard mix in methylene chloride, Sigma-Aldrich) injected under the same conditions.

For comparative purposes, commercial surfactin provided by Sigma Aldrich was also analyzed using the same methodology.

Proteins content in the biosurfactant extract was calculated by determining the $\mathrm{N}$ content of the extract. The sample was decomposed by combustion and then was analyzed using chromatographic with thermal conductivity detection (TCD) in a Carlo Erba EA-1108CHNS-O element analyzer. The value of $\mathrm{N}$ was transformed into protein content multiplying by 6.25 [9].

\subsection{Resin preparation}

The resins used in this work consisted of Amberlite IRA 400 (anionic resin) and Amberlite IR 120 (cationic resin). The main characteristics of these resins are included

in Table 1. Before utilization, Amberlite IRA 400 was converted into its $\mathrm{OH}^{-}$form by washing the resin sequentially with $1 \mathrm{~N} \mathrm{NaOH}$ solution and distilled water (until $\mathrm{pH}=7$ ); whereas Amberlite IR 120 was converted into its $\mathrm{H}^{+}$form by washing it sequentially with $1 \mathrm{~N} \mathrm{HCl}$ solution and distilled water (until $\mathrm{pH}=7$ ). Following resins were air dried during 24h, until a humidity of 36\% for Amberlite IRA 400 and 42\% for Amberlite IR 120 was achieved. 


\subsection{Experimental design}

A Box-Behnken factorial design was applied to study the ionic properties of biosurfactant extracted from CSL [10]. Different ionic exchange experiments were carried out by diluting the biosurfactant solution (BS) 0,10 or 20 times and treated with different amounts of anionic (Amberlite IRA 400) or cationic resin (Amberlite IR 120). Ionic exchange experiments were carried out at room temperature, with a working volume of $10 \mathrm{~mL}$, using a batch operation system at $200 \mathrm{rpm}$. The $\mathrm{pH}$ of the biosurfactant extract obtained was 4.0 and it was adjusted to $\mathrm{pH} 5.0$, using $\mathrm{NaOH} 1 \mathrm{M}$, in order to obtain a more biocompatible solution, according to shampoos formulations. Furthermore, at different intervals of time, established by the statistical design, samples of the aqueous solutions were obtained, and centrifuged at $5000 \mathrm{rpm}$ during $30 \mathrm{~min}$. After that, the surface tension of samples was measured.

Table 2 includes the range of the independent variables studied. The standardized (coded) dimensionless independent variables used, with limits of variation $(-1,1)$, were defined as $X_{1}$ (dilution rate of BS), $X_{2}$ (amount of anionic or cationic resin) and $X_{3}$ (extraction time); whereas the dependent variables consisted of the surface tension of BS after treatment with the anionic or cationic resins.

The relationship between coded and un-coded variables was established by linear equations deduced from their respective variation limits, according to equation 1.

$$
X_{i}=\left(\frac{z_{i}-z_{i}^{0}}{\Delta z_{i}}\right) \beta_{d}
$$

where $\Delta z_{i}$ is the distance between the real value in the central point and the real value in the superior or inferior level of a variable; $\beta_{d}$ is the major coded limit value in the matrix for each variable, and $\mathrm{z}_{\mathrm{i}}^{0}$ is the real value in the central point. Coded variables were then assigned values of $-1,0$ and +1 , corresponding to the minimum, central and maximum limits of variation for each variable. The response surface obtained from the coded 
variables is, therefore, not influenced by the magnitude of each variable, which allows combination of the factors on a dimensionless scale.

For comparative purposes, the ionic behavior of surfactin was also determined. In this way, $0.5 \mathrm{~g}$ of the resin was mixture with $5 \mathrm{~mL}$ of a surfactin solution, during $30 \mathrm{~min}$ at room temperature. The concentration selected was its $\mathrm{CMC}(10 \mathrm{mg} / \mathrm{L})$, in order to be able to see changes on surface tension. Thus, the conditions established are between the ranges studied during this experimental design.

\subsection{Statistical analysis}

The experimental data were analyzed by the Response Surface method with Statistica 7.0 software, by adjusting the dependent experimental data obtained to a quadratic function (equation 2); where $Y$ is the dependent variable, $\beta$ denotes the regression coefficients (calculated from experimental data by multiple regressions using the leastsquares method) and $X$ denotes the independent variables.

$$
\begin{aligned}
Y=\beta_{0}+\beta_{1} X_{1} & +\beta_{2} X_{2}+\beta_{3} X_{3}+\beta_{12} X_{1} X_{2}+\beta_{13} X_{1} X_{3}+\beta_{23} X_{2} X_{3}+\beta_{11} X_{1}^{2}+\beta_{22} X_{2}^{2} \\
& +\beta_{33} X_{3}^{2}
\end{aligned}
$$

\subsection{Determination of ionic behavior of biosurfactant}

The ionic charge of the biosurfactant can be established based on its affinity to interact with the anionic or cationic resins. Therefore, in the case that the biosurfactant is amphoteric, the dominant charge of the biosurfactant will be established following equation 3.

$$
Y_{3}=Y_{1} / Y_{2}
$$

where $Y_{3}$ measure the preference of the biosurfactant to act as an anionic or cationic biosurfactant; whereas $Y_{1}$ and $Y_{2}$ will be the surface tension of BS aqueous solution in presence of the anionic or cationic resins respectively, under the same operational 
conditions. If $Y_{3}>1$ the biosurfactant will have a dominant anionic charge; whereas if $Y_{3}<1$ the biosurfactant will have a dominant cationic charge.

\section{Results and discussion}

\subsection{Validation of the biosurfactant from CSL}

The biosurfactant extracted in this work from CSL, at concentrations higher the CMC, which is $200 \mathrm{mg} / \mathrm{L}$, was able to reduce the surface tension of water up to $37.23 \pm 0.12$ $\mathrm{mN} / \mathrm{m}$. Figure 1 shows the spectrum obtained from fatty acid analysis, using a FAMEs mix standard (Fig. 1A), compared with the spectrum of a commercial biosurfactant, produced by Bacillus subtilis named surfactin (Fig. 1B), and the fatty acid spectrum of the biosurfactant extracted from CSL and subjected at evaluation in this work (Fig. 1C). It can be observed that the three main fatty acids present in surfactin, which are the Hexadecanoic acid-methyl ester, Methyl stearate and the 9-Octadecenoic acid (Z)methyl ester, are also in the biosurfactant extract from CSL. Furthermore, in Table 3 it can be observed that 9-Octadecenoic acid (Z)- methyl ester, not only is in both biosurfactants but there is the same proportion (21\%). It was noticed that the composition of the biosurfactant extracted from CSL was quite homogeneous, in comparison with the fatty acids found in other biosurfactant extracts obtained in previous works from CSL $[7,8]$.

The biosurfactant extract obtained directly from CSL constitute an interesting alternative to other biosurfactants reported in the literature like surfactin. In addition, once biosurfactant it is extracted, Vecino and coworkers [8] have proved that CSL keeps its nutritional properties as nutritional supplement.

The yield of biosurfactant obtained in this work was $5.60 \mathrm{~g}$ of biosurfactant $/ \mathrm{Kg}$ of CSL, which is in concordance with those reported in previous work [8]. The protein concentration of biosurfactant was about $11 \%$. It can be deduced because of the low 
value of protein, that probably the biosurfactant will be formed by low chains of amino acids forming different peptide chains.

Therefore, once it has been corroborated that CSL is a source of biosurfactants, the following step is to propose the best use for surface-active agents at industrial scale. An interesting purpose could be its application in the cosmetic and personal care industry. In these industries, as it was said, different kinds of surface-active compounds are included in different formulations depending on its ionic characteristics.

\subsection{Ionic behaviour study of the biosurfactant from CSL}

In the current work a design was established in order to study the ionic behaviour of the biosurfactant extracted from CSL. The utilization of incomplete factorial design like that proposed by Box-Behnken allows analysing the synergic effect between different independent variables established in a specific process. The independent variables selected in the study will allow analysing the effect of biosurfactant dilution rate on their ionic behavior and its interaction with the extraction time and resin amount. It can be speculated that the dilution rate applied to a biosurfactant can determine its ionic behaviour because, close to the $\mathrm{CMC}$, they form micelles and only the hydrophilic moiety of biosurfactant is in contact with the aqueous molecules.

The experimental test conditions (expressed as coded variables) and the experimental data obtained for the dependent variables $Y_{1}$ and $Y_{2}$, corresponding to the surface tension of BS solution, after treatment with Amberlite IRA 400 and Amberlite IR 120 respectively, are shown in Table 4.

Among the three independent variables included in the study the dilution rate of biosurfactant solution $\left(X_{1}\right)$ was the most significant for both resins as it can be observed in the pareto chart of standardized effects for the assays carried out with Amberlite IRA 400 (Fig. 2A) and with Amberlite IR 120 (Fig. 2B). When the data are analysed 
following the behavior of biosurfactant solution in presence of Amberlite IRA 400 it is observed that the second independent variable in order of importance is $\left(X_{2}\right)$, corresponding to the amount of resin, being the less significant variable, in this case, the contact time between the resin and the BS solution $\left(X_{3}\right)$. Regarding the assays carried out with Amberlite IR 120 it was observed that the most significant independent variable was the dilution rate of BS solution $\left(X_{1}\right)$, followed by the extraction time $\left(X_{3}\right)$, being the less significant variable $X_{2}$ (amount of resin).

Additionally, Table 5 shows the regression coefficients obtained for the dependent variables $Y_{1}$ and $Y_{2}$, as well as their statistical significance. Taking into account the pvalue, only those coefficients with $p$-value $<0.05$ were considered significant. The $p$ value is a function of the experimental results obtained for $Y_{1}$ and $Y_{2}$, relative the statistical model, established using the coefficients included in Table 5. Therefore, pvalue measure the probability of obtaining a theoretical result equal to that observed in the experimental assay by using the equations calculated by the model for $Y_{1}$ and $Y_{2}$. According to the p-values obtained, equations 4 and 5 can be used to predict the surface tension achieved in the BS aqueous solution after contacting with IRA 400 or IR 120 respectively, in the range established for the independent variables $X_{1}, X_{2}$ and $X_{3}$. In these equations only were included those significant coefficients with $\mathrm{p}<0.05$.

$$
\begin{gathered}
Y_{1}=\text { 71.37. }+5.36 X_{1}+1.21 X_{2}-2.44 X_{1} X_{3} \\
-5.73 X_{1}^{2} \\
Y_{2}=71.98+5.78 X_{1}-0.61 X_{2}+0.86 X_{3}+1.66 X_{1} X_{2}-2.68 X_{1} X_{3} \\
-6.53 X_{1}^{2}-0.49 X_{3}^{2}
\end{gathered}
$$


Figure 3 and Figure 4 shows the variation in the surface tension of BS aqueous solution, for Amberlite IRA 400 ad Amberlite IR 120 respectively, with the most significant independent variables. Therefore Figure 3 shows the variation in the surface tension of BS solution with the dilution rate $\left(X_{1}\right)$ and Amberlite IRA 400 amount $\left(X_{2}\right)$, fixing the no significant variable, $X_{3}$, at the lowest (Fig. 3A), intermediate (Fig. 3B) and highest value (Fig. 3C).

When the BS solution was concentrated at the CMC the resin entrapped only part of the biosurfactant whereas when it was diluted 10 times below the $\mathrm{CMC}$ it was observed that the resin Amberlite IRA 400 was able to entrap almost all the biosurfactant deduced by the surface tension of water after the treatment, even at the lowest resin amount. Moreover, it was noticed that the anion exchange between resin and the biosurfactant was very fast, in $2 \mathrm{~min}$ can be reached the equilibrium, therefore the differences between Figure 3A, Figure 3B and Figure 3C are negligible as it can be observed.

On the other hand, when the assays were carried out with Amberlite IR 120 the most significant variable was the dilution rate of BS $\left(X_{1}\right)$, followed the extraction time $\left(X_{3}\right)$, being the amount of resin $\left(X_{2}\right)$ the less significant variable. Figure 4 shows the variation in the surface tension with the dilution rate of BS solution $\left(X_{1}\right)$ and extraction time $\left(X_{3}\right)$, fixing the less significant variable, amount of Amberlite IR $120\left(X_{3}\right)$, at the lowest (Fig. 4A), intermediate (Fig. 4B) and highest value (Fig. 4C). Again it was observed a fast ionic exchange, but in this case the ionic exchange is cationic, observing an increase in the surface tension after 2 min of starting the cationic exchange assays.

Comparing the assays with both resins it can be observed that both, cationic and anionic resins, can remove and entrap the biosurfactant from the aqueous solution achieving a surface tension similarly to the pure water. Therefore, the data reveal that the biosurfactant extracted from CSL is amphoteric. 
Reasonable agreement was observed between the experimental and theoretical data predicted by equations 1 and 2 for the dependent variables assayed, $Y_{1}$ and $Y_{2}$, with $\mathrm{r}^{2}$ values of 0.95 and 0.98 respectively. Figure 5A and Figure 5B shows the agreement between experimental and theoretical data for variables $Y_{1}$ and $Y_{2}$ respectively.

During the experiments carried out with surfactin, negligible adsorption was observed either with Amberlite IRA 400 or Amberlite IR 120. Therefore, it can be deduced that surfactin is a non ionic biosurfactant.

Analysing the data from a biochemical point of view, it has been proved that biosurfactant obtained from CSL is mainly composed of fatty acids (as it can be observed in Fig. 1C and Table 3), which are a group of negatively charged substances and their negative charge is located at the end of the carboxyl groups, although once esterified they do not have charge. On the other hand, the hydrophilic moiety of lipopeptide biosurfactants can be nonionic or charged positively, negatively or even both, depending on the amino acids nature that compromise the biosurfactant. Hence, in the biosurfactant extracted form CSL, the charge of the biosurfactant will depend on the amino acids composition. There are 20 different amino acids most commonly occurring in nature and the charged amino acid residues include lysine $(+)$, arginine $(+)$, aspartate (-) and glutamate (-). Besides, based on the affinity of the amino acid chain for polar solvents, like water, they may be classified as hydrophobic (non polar) or hydrophilic (polar). Charged amino acids are also polar and therefore hydrophilic. The biosurfactant extracted from CSL is soluble in water probably due to the presence of polar amino acids that it is in concordance with the affinity of this biosurfactant for cationic and anionic resins. Therefore, it can be speculated that biosurfactant extracted form CSL is composed by lysine or arginine, that provide it with positive charge and aspartate and glutamate, that give to the biosurfactant also a negative charge. It is important 
differentiate glutamate from glutamine. Glutamate is not an essential amino acid that has a negative charge, while glutamine is a conditional amino acid that does not have a charge at all.

Based on the amino acid chains, most of the lipopeptide biosurfactants reported in the literature, are anionic or nonionic. Although a high variation, in the amino acid composition, even between biosurfactants, produced by the same microorganism, has been observed. Kowall et al. [11], have reported that Bacillus subtilis could produce 44 different types of surfactins depending on the amino acids that compound their hydrophilic moiety. Coronel-León et al. [12], have reported that the biosurfactant produced by Bacillus licheniformis is composed by a peptide moiety consisting of glutamine as the N-terminal amino acid, two leucines, valine, aspartic, leucine and isoleucine as the C-terminal amino acid; whereas, surfactin, the first biosurfactant reported in the literature and produced by $B$. subtilis, was composed by glutamic acid, 4 leucines, one valine and aspartic acid [13-15]

Moreover, some authors [16] studied the effect of media composition on the production of amino acids by different microorganisms, observing that CSL induced the production of lysine, glutamic acid, aspartic acid and alanine. This effect is in concordance with the results obtained in this work, where it was observed that the lipopeptide biosurfactant extracted from CSL is amphoteric and it could be composed by lysine $(+)$ and/or arginine (+), aspartate (-) and/or glutamate (-). Following with this theory, Van Walsem and Thompson [17] have also observed that CSL is a good protein source to produce lysine by Corynebacterium glutamicum.

Additionally, in order to measure the dominance of the anionic or cationic character of the biosurfactant extracted from CSL, a new variable $\left(Y_{3}\right)$ was included in Table 4 . This variable was calculated based on the relationship between $Y_{1}$ and $Y_{2}$, following 
equation 3. Variables $Y_{1}$ and $Y_{2}$ measure the surface tension of BS solution in presence of the anionic or cationic resins respectively, therefore that resin which produce a higher increment in the surface tension of BS solution will define the character of the biosurfactant under the conditions used in this study ( $\mathrm{pH}=5.0$ and room temperature). The values obtained for $Y_{3}$ shows that there is no a clear charge predominance, since under all the conditions assayed, the relationship between $Y_{1}$ and $Y_{2}$ is close to 1 .

Contrarily, to the biosurfactant evaluated in this work, the biotechnological production of biosurfactants, like surfactin, for industrial applications is not cost competitive, limited by its high cost production and its low yields [18]. In this case, the biosurfactant, obtained from CSL, has "zero" production cost, because it is obtained spontaneously as a sub-product in an industrial stream, being the only cost that it is necessary to afford for its industrial, derive from the separation and purification operations.

From de results achieved in this work, it can be deduced that the lipopeptide biosurfactant, extracted from CSL, is composed by terminal polar amino acids, charged negatively and positively, which is not very common, because only 4 charged amino acids exist in the nature. For this reason, it can be indistinctly entrapped by anionic and cationic resins. Moreover, this biosurfactant is soluble in water what is in agreement with its amphoteric character, making it particularly suited for use in personal care preparations over sensitive skins.

\section{Acknowledgements}

This study was supported by the Spanish Ministry of Economy and Competitiveness under the project CTM2015-68904 (FEDER funds), as well as X. Vecino is grateful for her Juan de la Cierva contract (FJCI-2014-19732). Also, L. Rodríguez-López acknowledges to the Xunta de Galicia (Spain) for her pre-doctoral fellowship (ED481A2016/006). 


\section{References}

[1] Miyake M, Kakizawa YJ (2010) Morphological study of cationic polymer-anionic surfactant complex precipitated in solution during the dilution process. J Cosmet Sci 61: $289-301$

[2] Leidreiter HI, Grüning B, Kaseborn D (1997) Amphoteric surfactants: Processing, product composition and properties. J Cosmet Sci 19: 239-253

[3] Blagojević SN, Blagojević SM, Pejić ND (2016) Performance and Efficiency of Anionic Dishwashing Liquids with Amphoteric and Nonionic Surfactants. J Surfactants Deterg 19: 363-372

[4] Corazza M, Lauriola MM, Bianchi A, Zappaterra M, Virgili A (2010) Irritant and sensitizing potential of eight surfactants commonly used in skin cleansers: An evaluation of 105 patients. Dermatitis 21: 262-268

[5] De S, Malik S, Ghosh A, Saha R, Saha B (2015) A review on natural surfactants. RSC Advances 5: 65757-65767

[6] Sekhon BS (2013) Surfactants: pharmaceutical and medicinal aspects. J Pharm Technol Res Manag 1:11-36

[7] Vecino X, Barbosa-Pereira L, Devesa-Rey R, Cruz JM, Moldes AB (2014) Study of the surfactant properties of aqueous stream from the corn milling industry. J Agric Food Chem 62:5451-5457

[8] Vecino X, Barbosa-Pereira L, Devesa-Rey R, Cruz JM, Moldes AB (2015) Optimization of liquid-liquid extraction of biosurfactants from corn steep liquor. Bioproc Biosyst Eng 38:1629-1637

[9] Mariotti F, Tomé D, Mirand P (2008) Converting nitrogen into protein--beyond 6.25 and Jones' factors. Crit Rev Food Sci Nutr 48:177-184 
[10] Box GEP, Behnken DW (1960) Some new three level designs for the study of quantitative variables. Technometrics 2:455-475

[11] Kowall M, Vater J, Kluge B, Stein T, Franke P, Ziessow D (1998) Separation and characterization of surfactin isoforms produced by Bacillus subtilis okb 105. J Colloid Interface Sci 204:1-8

[12] Coronel-León J, de Grau G, Grau-Campistany A, Farfan M, Rabanal F, Manresa A, Marqués AM (2015) Biosurfactant production by AL 1.1, a Bacillus licheniformis strain isolated from Antarctica: production, chemical characterization and properties. Ann Microbiol 65: 2065-2078.

[13] Arima K, Kakinuma A, Tamura G (1968) Surfactin, a crystalline peptide-lipid surfactant produced by Bacillus subtilis, isolation, characterization and its inhibition of fibrin clot formation. Biochem Biophys Res Commun 31: 488-494.

[14] Kakinuma A, Hori M, Isono M, Tamura G (1969) Determination of amino acid sequence in surfactin, a crystalline peptide lipid surfactant produced by Bacillus subtilis. Agric Biol Chem 33: 971-972

[15] Liu XY, Yang SZ, Mu BZ (2005) Molecular structures of microbial lipopeptides. Biotechnol Bull 4:18-26

[16] Riaz S, Shakoori AR (2002) Effect of media composition on the production of amino acids through microbial fermentation by utilizing agricultural wastes. J Zool 34: $325-330$

[17] Van Walsem HJ, Thompson MC (1997) Simulated moving bed in the production of lysine. J Biotechnol 59: 127-213

[18] Chen WC, Juang RS, Wei YH (2015) Applications of a lipopeptide, surfactin, produced by microorganisms. Biochem Eng J 103: 158-169. 
L. Rodríguez-López is a PhD student in the Chemical Engineering Department at University of Vigo and her research involves the purification and characterization of biosurfactants obtained by biotechnology processes as well as their application on the cosmetic field.

M. Rincón-Fontán is a $\mathrm{PhD}$ student in the Chemical Engineering Department at University of Vigo and her studies involved the formulation and application of cosmetic and personal care products such as shampoos, creams among others, using the biosurfactant obtained from corn milling industry.

X. Vecino is a $\mathrm{PhD}$ in the Chemical Engineering Department at the Universitat Politècnica de Catalunya and her researcher focuses on the utilization of agro industrial wastes or low-cost materials like zeolites as adsorbents for ion exchange/adsorption removal processes in batch and column modes.

A.B. Moldes is an Associate Professor of the Chemical Engineering Department at the University of Vigo and her research is based on the biotechnological production of natural compounds, such as biosurfactants, bioemulsifiers, lactic acid, among others, by using renewable carbon sources, for application in the pharmaceutical, biomedical, environmental and food industries.

J.M. Cruz is a Full Professor and the Director of the Chemical Engineering Department at University of Vigo. He has a huge number of publications about the utilization of agro-industrial residues as well as on the production of high-value compounds like biosurfactants and antioxidants, and their application in the food, pharmaceutical and environmental industry. 